\title{
Análises físicas das águas subterrâneas de poços localizados nas comunidades Várzea Comprida dos Oliveiras e Bezerro no município de Pombal-PB
}

\section{Analysis of physical well located in groundwater communities Várzea Comprida dos Oliveiras $e$ Bezerro in the municipality of Pombal-PB}

\author{
Alan Dél Carlos Gomes Chaves ${ }^{1}$, Ricardo Ricelli Pereira de Almeida ${ }^{2}$, Manoel Moisés Ferreira de Queiroz ${ }^{3}$, Francisco Tales \\ da Silva ${ }^{4}$ Elton Pereira da Silva.
}

\begin{abstract}
RESUMO - As águas subterrâneas são fundamentais para o desenvolvimento humano. No Brasil, elas desempenham importante papel no abastecimento público e privado, suprindo as mais variadas necessidades de água em diversas cidades e comunidades, bem como em sistemas autônomos residenciais, indústrias, serviços, irrigação agrícola e lazer. As águas subterrâneas são aquelas que se infiltram através da superfície do terreno e enchem os espaços vazios dos interstícios das rochas. A irrigação é uma prática agrícola que visa principalmente atender as necessidades hídricas das culturas no momento adequado. A água utilizada na irrigação das hortaliças é derivada de poços tubulares e do rio Piranhas. O trabalho teve como objetivo avaliar os parâmetros físicos da água dos poços das comunidades Bezerro e Várzea Comprida dos Oliveiras. A pesquisa foi desenvolvida no município de Pombal-PB, por meio de visitas feitas nas comunidades, as quais trabalham com a produção de hortaliças irrigada com água subterrânea. Foram coletadas 18 amostras de água nas comunidades citadas e realizadas as seguintes análises físicas: Turbidez, temperatura e cor. Os poços apresentaram redução nos valores de Turbidez a partir de fevereiro, pois as chuvas conseguiram diluir os materiais presentes na água dos poços. Os valores obtidos para cor estão dentro do padrão exigido para irrigação. A temperatura apresentou pouca oscilação nos valores no decorrer do trabalho.
\end{abstract}

Palavras - Chave - Qualidade da água, Turbidez, Água de irrigação, Cor e Temperatura.

\begin{abstract}
Groundwater is fundamental for human development. In Brazil, they play an important role in the public and private supply, supplying the various water needs in various cities and communities as well as in residential autonomous systems, industries, services, agricultural irrigation and recreation. Groundwater is those that seep through the ground surface and fill the empty spaces of the interstices of the rocks. Irrigation is an agricultural practice that aims primarily to meet the water needs of crops at the right time. The water used for irrigation of vegetables is derived from wells and the Piranhas river. The study aimed to evaluate the physical parameters of the water from the wells of communities Bezerro e Varzea comprida dos Oliveiras. The research was conducted in the municipality of Pombal-PB, through visits made to the communities they work with the production of vegetables irrigated with groundwater. We collected 18 samples of water in the said communities and made the following physical analysis: Turbidity, temperature and color. He wells showed reduction in turbidity values from February as rains failed dilute the material in the water of the wells. The values obtained for the color are within the required standard for irrigation. The temperature showed little fluctuation in values during the work.
\end{abstract}

Key words: Water quality, turbidity, water for irrigation, color and temperature.

\footnotetext{
*Autor para correspondência

Recebido para publicação em 07/11/2014; aprovado em 26/05/2015

${ }^{1}$ Mestre em Sistemas Agroindustriais - UFCG; Fone: (83) 96589908; E-mail: alan.delcarlos@ hotmail.com

${ }^{2}$ Mestrando em Sistemas Agroindustriais - UFCG; E-mail: ricelli2008@bol.com.br

${ }^{3}$ Doutor em Engenharia Hidráulica e Saneamento - USP 2002; E-mail: moises@ ccta.ufcg.edu.br

${ }^{4}$ Graduado em Geografia - CFP/UFCG; E-mail: t.silva_@outlook.com

${ }^{5}$ Graduando em Psicologia pela Universidade Santa Maria de Cajazeiras-PB; E-mail: helthonsilvasuporte@hotmail.com
} 


\section{INTRODUÇÃO}

Dos recursos naturais importantes, a água é o que possui grande destaque, pois sua disponibilidade e acesso são necessários a todo tipo de vida no planeta, bem como para a maioria dos meios de produção (SARDINHA et al.,2008).

Dessa forma, alimentos que entram em contato com águas contaminadas e são consumidos crus, constituem fontes prováveis de microrganismos (PACHECO et al. 2002). Os principais agentes biológicos encontrados nessas águas são as bactérias patogênicas, os vírus e os parasitos. Esses organismos encontrados na água ou nos alimentos representam uma das principais fontes de morbidade no país, sendo responsáveis por inúmeros casos de doenças infecciosas, parasitárias, enterites e diarreias infantis, podendo levar até os indivíduos a morte (ALMEIDA FILHO, 2008).

O monitoramento de parâmetros de qualidade da água constitui-se em ferramenta básica para avaliar alterações ambientais causadas pela ação antrópica (MOLOZZIET al., 2006).

Os Guias da Organização Mundial da Saúde (OMS) têm sido utilizados como referência mundial na orientação dos padrões e nas legislações nacionais referentes à qualidade da água para consumo humano. Usualmente tem exercido grande influência na elaboração e atualização da legislação brasileira sobre potabilidade (HELLER et al., 2005).

Os impactos ambientais nos recursos hídricos gerados pelas atividades agrícolas, não podem ser dissociados dos impactos nas próprias áreas de produção, devendo seu monitoramento e as medidas preventivas, estarem sempre integradas de uma forma sistêmica (ANDREOLI, 1993).

De acordo com o exposto acima, o trabalho teve como objetivo verificar as características físicas das águas de poços tubulares usadas na irrigação de Hortaliças nas comunidades Bezerro e Várzea comprida dos Oliveiras.

\section{MATERIAL E MÉTODOS}

O presente trabalho de campo foi desenvolvido no município de Pombal - PB, através de visitas feitas nas comunidades de Várzea Comprida dos Oliveiras e Bezerro as quais trabalham com a produção de hortaliças irrigadas com água subterrânea de poços tubulares, onde foram coletadas 18 amostras de água para análises físicas, como por exemplo: Turbidez, Cor e Temperatura foram analisadas in loco .

A pesquisa foi desenvolvida em três etapas. $\mathrm{Na}$ primeira etapa, foi feito um reconhecimento da influência do projeto, através de visitas aos dos produtores na comunidade, na qual foi feito também levantamento dos poços artesianos existentes na comunidade Várzea Comprida dos Oliveiras e na comunidade Bezerro e na segunda etapa foi feito levantamento das culturas agrícolas desenvolvidas na região. A terceira etapa foi realizada através do georreferenciamento e definição dos poços tubulares.

A área em estudo da pesquisa está situada na Mesorregião do Sertão e Microrregião Homogênea de Sousa, no Estado da Paraíba, situado na região nordeste do Brasil. O município é um dos mais antigos do estado da Paraíba, e possui a segunda maior área territorial do estado da Paraíba em extensão territorial possuindo $889 \mathrm{~km}^{2}$. Segundo o IBGE
(Instituto Brasileiro de Geografia e Estatística), a população em 2010 era estimada em 32.143 habitantes.

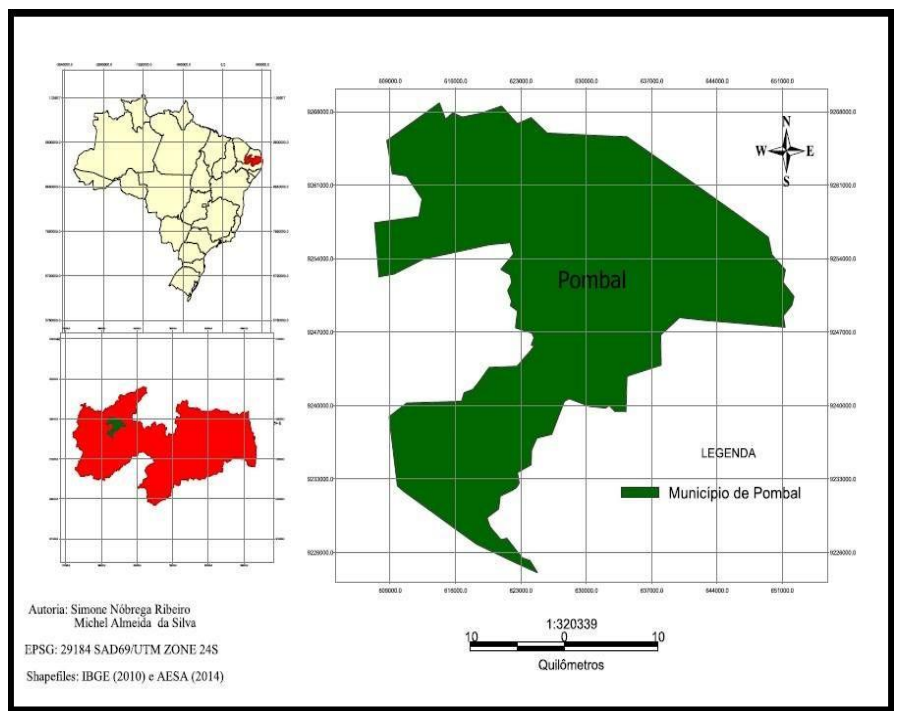

Figura 1: Localização do Município de Estudo

Fonte: Ribeiro e Silva, 2015.

Os pontos de coleta de água foram georreferenciados através do aparelho de GPS e transferidos para localização em mapa com os dados de localização geográfica, que permitiu a obtenção dos pontos com latitude e longitude em projeção UTM (Universal Transversa de Mercator).

De acordo com os dados coletados através das entrevistas, foram observados problemas como escassez de água, prejudicando as atividades agrícolas, devido o baixo nível de água do rio e dos poços.

As amostras de água foram coletas em fonte natural; em poços tubulares, no período de seca do ano de 11/2013 $05 / 2014$. Nas comunidades em estudo foram contatados os Agricultores e a Presidente da Associação de Produtores Rurais.

As amostras foram coletadas com coletor do tipo: garrafa pet de plástico, com diâmetro de 10 e comprimento de $22 \mathrm{~mm}$.

Todos os procedimentos de coleta, preparo dos frascos de coleta, transporte e acondicionamento das amostras destinadas as análises físico-químicas seguirão as recomendações do "Standard Methods for the Examination of Water and Wastewater 20TH ed." (APHA, 1998).

As amostras foram acondicionadas em caixas de isopor e preservadas em gelo até a chegada ao Laboratório de Análise de Solo e Água, do Instituto Federal de Educação, Ciência e Tecnologia (IFPB-Sousa).

Nas amostras coletadas foram analisados: turbidez, temperatura e cor.

As coletas foram realizadas sempre no período da manhã, sempre no mesmo ponto de referência (fonte de água). Coletou sempre $100 \mathrm{~mL}$ da amostra em frasco para analise in loco e outro frasco de 2 litros para analise em laboratório, previamente preparados.

\section{RESULTADOS E DISCUSSÃO}

Entre os poços da comunidade Bezerro, o poço B foi o que apresentou os maiores resultados, com valores de 7,34 e $6,33 \mathrm{mg} \mathrm{Pt} \mathrm{/} \mathrm{L} \mathrm{entre} \mathrm{07/02/2014} \mathrm{a} \mathrm{17/04/2014}$ 
respectivamente. Para os poços da comunidade da várzea comprida da Oliveiras, os maiores valores foram encontrados na coleta de 16/01/2014 (figura 2A e B), com o poço J apresentando $2,55 \mathrm{mg} \mathrm{Pt} / \mathrm{L}$.

Os poços $\mathrm{F}$ e E apresentaram os menores valores na data de 26/03/2014 (0,18 a 0,23 mg Pt / L). Resultados semelhantes foram observados por Barcellos et al., (2000) e Silva, Araújo e Souza (2007) estudando a turbidez de poços rasos no município de Lavras-MG, onde encontraram valores de 2, $52 \mathrm{mg} \mathrm{Pt} / \mathrm{L}$ e $5 \mathrm{mg} \mathrm{Pt} / \mathrm{L}$.

Normalmente as águas subterrâneas são isentas de cor, mas de forma atípica podem atingir valores até $100 \mathrm{mg}$ $\mathrm{Pt} / \mathrm{L}$ (FEITOSA E FILHO, 1997) o que provoca uma repulsa psicológica pelo consumidor. Rocha (2008) avaliando a qualidade das águas dos poços do estado do Piauí, observou que a turbidez apresentou um valor máximo de $787 \mathrm{mg} \mathrm{Pt} \mathrm{/} \mathrm{L.}$

(A)

Tabela 1 - Medição do parâmetro Cor dos poços da Comunidade Várzea Comprida dos Oliveiras - Pombal/PB.

\begin{tabular}{ccc}
\hline Poço & Nome & Valor medido da cor g/L \\
\hline 1 & A & 0,006 \\
2 & B & 0,02 \\
3 & C & 0,00 \\
4 & D & 0,00 \\
5 & E & 0,01 \\
6 & F & 0,00 \\
7 & G & 0,00 \\
8 & H & 0,02 \\
9 & I & 0,006 \\
10 & J & 0,02 \\
11 & L & 0,02 \\
\hline
\end{tabular}

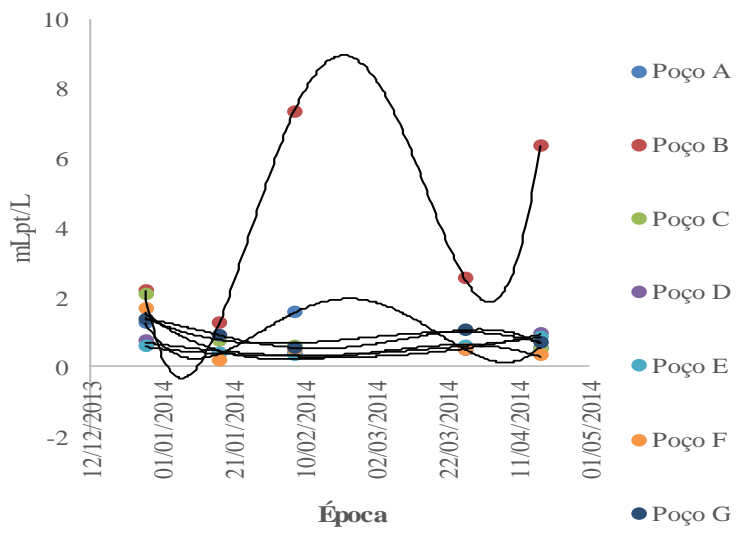

(B)

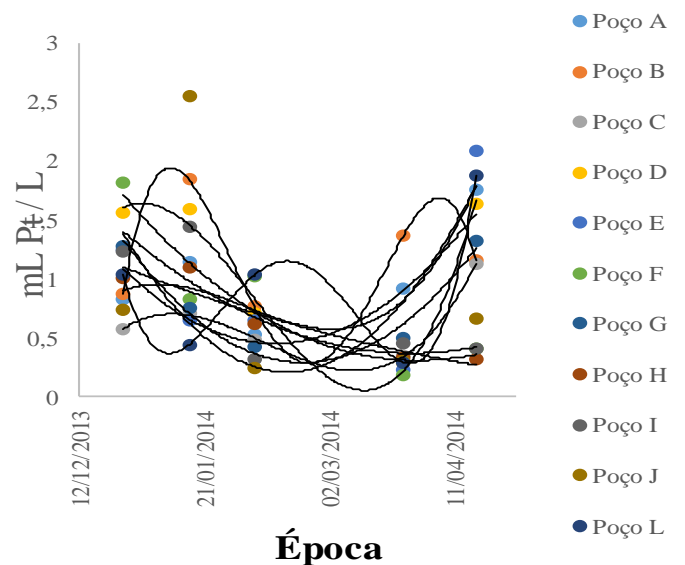

Figura 2: Turbidez das águas dos poços da comunidade Bezerros e Várzea comprida dos Oliveiras

O parâmetro cor para águas subterrâneas derivadas de poços tubulares usadas para fins de irrigação de hortaliças não é um parâmetro vigente em legislação, mas foi medido com a finalidade de analisar e comparar com o poço 2 que é o manancial usado para abastecimento humano das comunidades acima citadas.

Um trabalho desenvolvido por Barcellos et al., (2000) nas águas de mananciais do município de lavras-MG, verificaram valores de 5,2 de $\mathrm{mg} / \mathrm{L}$ para poços rasos. Resultados similares foram encontrados por Manchester et al., (2013) avaliando as águas de minas do município de Teófilo Otoni-MG, verificaram resultados próximos de $6 \mathrm{mg} / \mathrm{L}$. Nascimento e Barbosa (2005) encontraram valores de 6,4 mg / L nas águas da bacia do Caia no município de Salvador-BA.
Tabela 2 - Medição do parâmetro Cor dos poços da Comunidade Bezerro - Pombal/PB.

\begin{tabular}{ccc}
\hline Poço & Nome & Valor medido da cor g/L \\
\hline 1 & Horta & 0,04 \\
2 & Consumo & 0,06 \\
3 & B & 0,02 \\
4 & C & 0,02 \\
5 & D & 0,00 \\
6 & E & 0,04 \\
7 & F & 0,03 \\
8 & Rio Piranhas & 0,24 \\
\hline
\end{tabular}


(A)

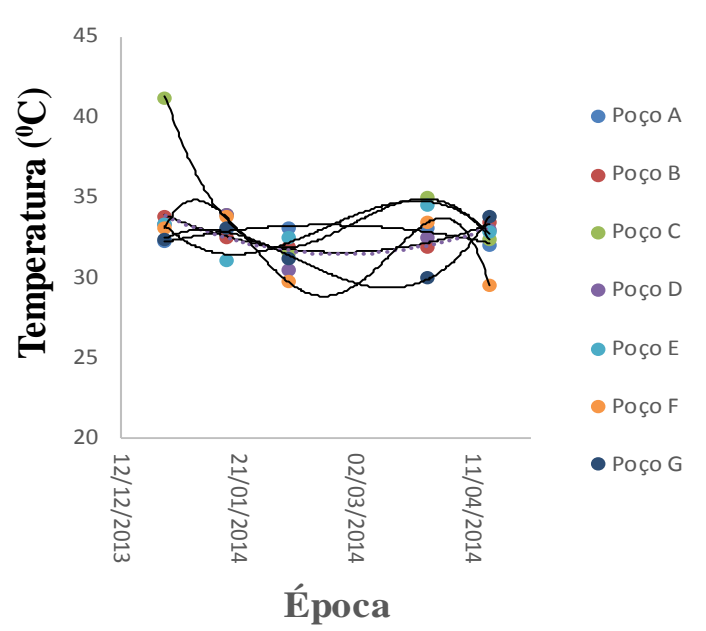

(B)

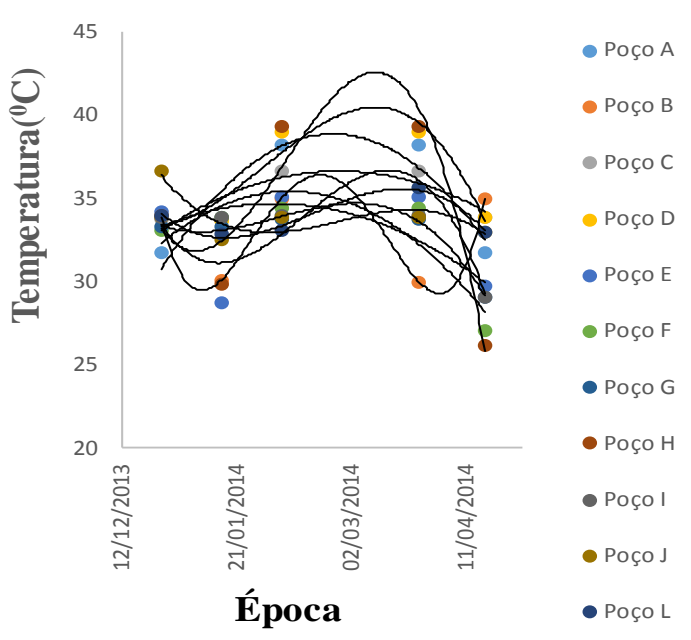

Figura 3: Temperatura média do ar nos poços das comunidades Bezerro e Várzea comprida das Oliveiras

A temperatura da água dos poços da comunidade Bezerro manteve valores constantes durante o período de monitoramento, com uma pequena redução mais acentuada no poço C (41,2 a $\left.33,9{ }^{\circ} \mathrm{C}\right)$ entre $27 / 12 / 2013$ a $17 / 01 / 2014$ e 27/03/2014 a 17/04/2014, já o poço G apresentou um aumento entre 27/03/2014 e 17/04/2014, mostrando valores de $\left(30\right.$ a $\left.33,7^{\circ} \mathrm{C}\right)$ (figura 4$)$.

Os maiores valores foram encontrados no início do monitoramento, com valor de $41{ }^{\circ} \mathrm{C}$ no poço C. Já os poços da comunidade várzea comprida da Oliveiras apresentaram os menores valores entre 26/12/2013 e 16/01/2014 e a partir de 26/03/2014 (figura 31).

$\mathrm{O}$ poço $\mathrm{D}$ foi o tratamento que obteve os maiores resultados, com média de $35{ }^{\circ} \mathrm{C}$. Os poços $\mathrm{E}$ e $\mathrm{F}$ demonstraram os menores valores médios de graus Celsius entre os poços $\left(3,54\right.$ e $\left.3,56{ }^{\circ} \mathrm{C}\right)$.

Em uma pesquisa desenvolvida por Barcellos et al. (2000) nas águas de poços rasos, em Lavras-MG, encontraram valores médios de $23{ }^{\circ} \mathrm{C}$. Resultados semelhantes foram encontrados por Nascimento e Barbosa (2005), onde eles avaliando a qualidade da água de um aquífero freático na cidade de Salvador, encontraram valor médio de 26.

\section{CONCLUSÃO}

Conclui-se que a água subterrânea dos poços tubulares usada na irrigação de hortaliças das comunidades de Várzea Comprida dos Oliveiras e Bezerro não apresentaram alterações significativas quanto a qualidade para essa atividade fim.

A cor não é normalmente um Parâmetro exigido para águas subterrâneas de poços tubulares utilizadas para irrigação de hortaliças.

Os poços apresentaram redução nos valores de Turbidez a partir de Fevereiro, pois as chuvas conseguiram diluir os materiais presentes na água dos poços.

Verificou-se que a temperatura variou pouco durante as medições realizadas in locu.

\section{REFERÊNCIAS BIBLIOGRÁFICAS}

Agência Nacional de Águas (ANA). 2005a. Panorama da qualidade das águas subterrâneas no Brasil. Brasília. Disponível em: http://www.ana.gov.br/sprtew/recursoshidricos.asp >

Agência Nacional de Águas (ANA). 2005b. Disponibilidade e demandas de recursos hídricos no Brasil. Brasília. Disponível em: http://www.ana.gov.br/sprtew/recursoshidricos.asp >

ANDREOLI, C.V. Influência da Agricultura na Qualidade da Água. Curitiba. OPS. 15 p, 1993.

ALMEIDA FILHO, P. C. de. Avaliação das condições ambientais e higiênico-sanitárias na produção de hortaliças folhosas no núcleo hortícula suburbano de Vargem Bonita, Distrito Federal. Dissertação. (Mestrado em Planejamento e Gestão Ambiental). Universidade Católica de Brasília, Brasília. 103p., 2008.

BARCELlOS, C. M. et al. "Avaliação da qualidade da água e percepção higiênico-sanitária na área rural de Lavras, Minas Gerais, Brasil, 1999-2000 Water quality evaluation in rural areas of Lavras, Minas Gerais, Brazil, 1999-2000." Cad. Saúde Pública 22.9 (2006): $1967-$ 1978.

HELLER, L. et al. Terceira edição do guias da Organização mundial da Saúde: queimpactoesperar na Portaria $\mathrm{n}^{\circ}$ 518/2004? In: CONGRESSO BRASILEIRO DEENGENHARIASANITÁRIA E AMBIENTAL, 23., 2005, Campo Grande, MS. Anais... Rio de Janeiro: ABES, 2005.

FEITOSA, F.A.C; FILHO, J.M: Hidrogeologia Conceitos e Aplicaçãoes: Noções de hidroquimica. CPRM, LABHID-UFPE, Fortaleza, 1997. p. 81-108

MANCHESTER R. S. A. S.; RODRIGUES, J. L.; BOMFETI, C. A.; GONÇALVES, A. M. P. M.; CARLOS, T. M.; 
Determinação da qualidade da água de Minas na área urbana do Município de Teófilo Otoni - MG Brasil. Revista Vozes do Vale, Murici-MG, UFVJM v.2, n.3, p.1-13, 2013.

MOLOZZIET, J.; PINHEIRO, A.; SILVA, M. R. da Qualidade da água em diferentes estádios de desenvolvimento do arroz irrigado. Pesquisa Agropecuária Brasileira, v.41, n.9, p.1393-1398, 2006.

NASCIMENTO, S. A. M.; BARBOSA, J. S. F. Qualidade da água do aquífero freático no alto cristalino de Salvador, Bacia do Rio Lucaia, Salvador, Bahia. Revista Brasileira de Geociências, v. 35, n. 4, p. 543-550, 2005

OLIVEIRA, C. A; GERMANO, P. M. Estudo da ocorrência deenteroparasitas em hortaliças comercializadas na região metropolitana de São Paulo, SP. Revista de Saúde Pública. v.26, p.283-289, 1992

PACHECO, M. A. S. R. et al. Condições higiênico-sanitárias de verduras e legumes comercializados no CEAGESP de Sorocaba - SP. Higiene Alimentar, São Paulo, v.16, n.101, p.50-55, 2002.

ROCHA, T. S.. Avaliação da qualidade das águas dos poços tubulares da bacia do Rio do Peixe equipados com dessalinizadores, com vistas ao aproveitamento econômico dos sais de rejeito. 96 f. 2008. Dissertação (Mestrado profissional em gerenciamento e tecnologias ambientais no processo produtivo) - Departamento de Engenharia ambiental (DEA)-Universidade Federal da Bahia, Salvador, 2008

SARDINHA, D. S.; CONCEIÇÃO, F. T.; SOUZA, A. D. G.; SILVEIRA, A.; DE JULIO, M.;GONÇALVES, J. C. S. I. Avaliação da qualidade da água e autodepuração do Ribeirão do Meio, Leme (SP). Revista Brasileira de Engenharia Sanitária e Ambiental, v.13, n.3,p.329$338,2008$.

SILVA, F. J. A. da, ARAÚJO, A. L. de, SOUZA, R. O. de. "Águas subterrâneas no Ceará: poços instalados e salinidade. Revista Tecnológica. Fortaleza. v. 28, n. 2, p. 136-159, dez. 2007.

SOUTO, R. A. de. Avaliação sanitária da água de irrigação e de alfaces (Lactuca sativa $l$.) produzidas no município de Lagoa Seca, Paraíba. Dissertação (Mestrado em Agronomia). Universidade Federal da Paraíba, Areia. 70 p., 2005 